\title{
Postparacentesis vulvar edema in ovarian hyper stimulation syndrome
}

\section{Sindhu Bhairavi*, Jisha Rajendran, Soumiyaroop Dash, Sebanti Dash}

Srishti Assisted Fertility and Advanced Laparoscopy Centre, Thattakutai, Moolakulam, Puducherry, India

Received: 22 August 2016

Revised: 17 September 2016

Accepted: 20 September 2016

\section{*Correspondence:}

Dr. Sindhu Bhairavi,

E-mail: sindhubhairavi86@gmail.com

Copyright: $\odot$ the author(s), publisher and licensee Medip Academy. This is an open-access article distributed under the terms of the Creative Commons Attribution Non-Commercial License, which permits unrestricted non-commercial use, distribution, and reproduction in any medium, provided the original work is properly cited.

\begin{abstract}
Ovarian hyper stimulation syndrome (OHSS) is a serious, potentially life threatening complication of ovulation induction. The clinical picture of severe OHSS includes ovarian enlargement, ascites, pleural effusion, increased coagulability, and electrolyte disorders. Very few cases of massive vulvar edema in OHSS have been reported in the literature.
\end{abstract}

Keywords: OHSS, Paracentesis Vulvar edema

\section{INTRODUCTION}

Ovarian hyperstimulation syndrome (OHSS) is a serious, potentially life threatening complication of ovulation induction. The clinical picture of severe OHSS includes ovarian enlargement, ascites, pleural effusion, increased coagulability, and electrolyte disorders. Very few cases of massive vulvar edema in OHSS have been reported in the literature. ${ }^{1,2}$

\section{CASE REPORT}

A 32-year-old woman was admitted to our hospital with OHSS and massive vulvar edema. She underwent ovarian stimulation with recombinant FSH 150 IU (Newmon-R, LG Life science, Jeollabuk, Korea) from D2 and recombinant LH 75 IU (Luveris, Merck serono, Switzerland) from D8 of menstrual cycle along with flexible GnRH antagonist protocol (Cetide, LG Life science, Uttarakhand, India). On day 11 of stimulation (E2 >3000 pg/mL), 250 micrograms of recombinant hCG (ovitrelle, Merck Serono, Modugno, Italy) was administered. 36 hours later, 16 follicles were aspirated. As p4 was $2.2 \mathrm{ng} / \mathrm{dl}$, frozen embryo transfer was planned. On $4^{\text {th }}$ day of HCG trigger, she was admitted with abdominal discomfort and abdominal pain that became severe during the next 3 days. She had hemoconcentration (PCV-44). She was treated conservatively with intravenous fluids, hestarch, analgesics and antibiotics. Because of increased respiratory difficulty and abdominal distension, she underwent transvaginal paracentesis. As significant reaccumulation of ascitic fluid occurred within 24 hours, she underwent repeat transvaginal paracentesis. Next day, she developed bilateral vulvar swelling confined to labia minora.

On $8^{\text {th }}$ day the labia had increased in volume to a thickness of 7-8 cm, with a tense skin (Figure 1). There were no signs of venous or lymphatic obstruction, peripheral edema, trauma, or allergic reaction. Ultrasound scan revealed enlargement of the ovaries (maximum diameter of left ovary, $104 \mathrm{~mm}$; right ovary, $80 \mathrm{~mm}$ ) and ascites. Laboratory tests showed PCV 38\%, hypoproteinemia (total protein levels, $1.6 \mathrm{gm} / \mathrm{dl}$; albumin levels, $1.4 \mathrm{gm} / \mathrm{dl})$.

The patient was treated with I v albumin. Local treatment consisted of ice packs and hydrocortisone and Gentamycin ointment alternatively. 


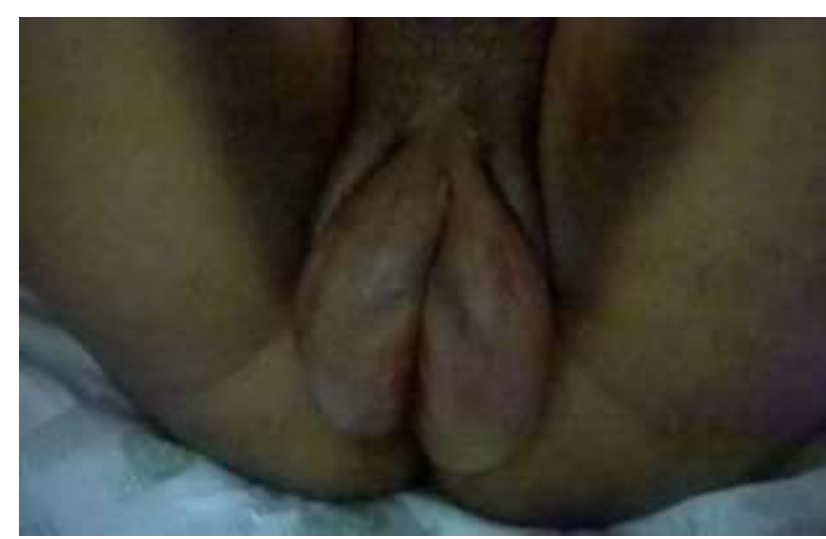

Figure 1: Massive vulvar edema 24 hours after Paracentesis.

Progressive abatement of symptoms occurred, and the vulvar edema resolved completely within 4 days. After treatment, total serum protein levels increased to $6.3 \mathrm{~g} / \mathrm{dl}$ and albumin levels increased to $4.4 \mathrm{~g} / \mathrm{dl}$ (Figure 2 and Figure 3).

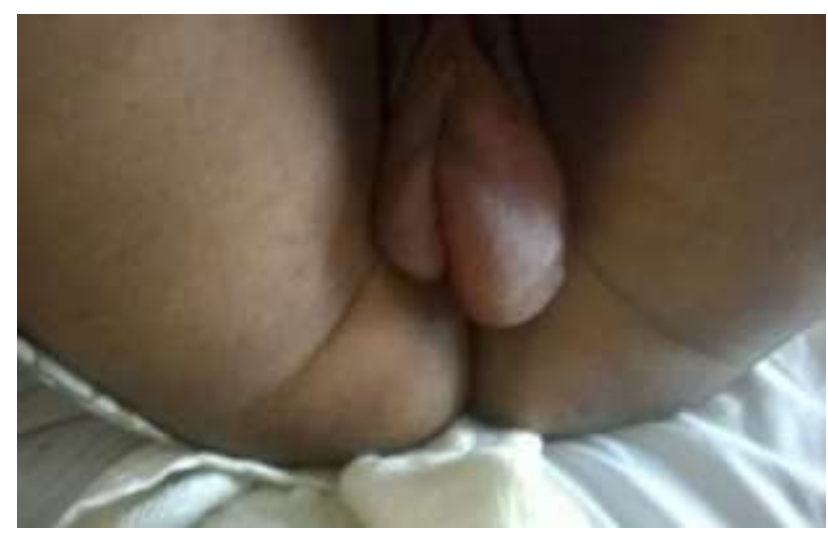

Figure 2: Day 2 of treatment.

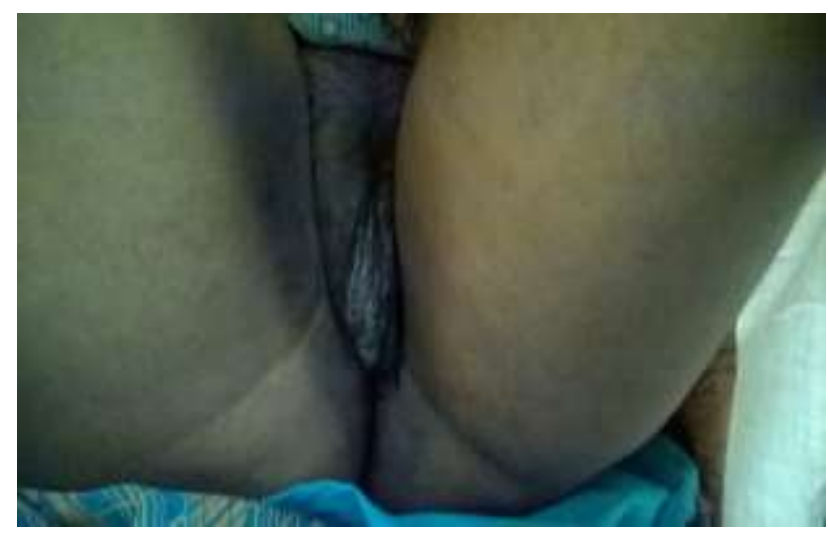

Figure 3: Day 4 of treatment.

\section{DISCUSSION}

Vulvar edema has been observed during pregnancies complicated by preeclampsia and after tocolysis for preterm labor. $^{1,2}$
Massive vulvar edema in association with OHSS was described for the first time in $1995 .^{3}$ The vulvar edema was treated with local application of cortisone, antibiotic ointment, and ice packs. According to the authors, the edema was the result of decreased oncotic and increased hydrostatic pressure due to OHSS.

A different hypothesis regarding the mechanism of vulvar edema was suggested by Cline et al. This condition, according to the author, occurs because the patients almost certainly have a patent canal of Nuck.

In a paper published in 1996, nine cases with severe OHSS that required abdominal paracentesis were described. ${ }^{5}$ Seven of them, in which the lower abdomen was punctured, developed within 10-24 hours, with unilateral vulvar edema corresponding to the puncture site. This phenomenon did not occur in the two patients who had punctures through the abdominal hypochondriac regions. The vulvar edema resolved over the subsequent 10 days.

The authors postulated that the fistula caused by a large bore needle between the peritoneum and abdominal subcutaneous tissues, along with the increased intraabdominal pressure due to ascites, may facilitate the passage of fluid and the development of ipsilateral edema in the labia, which are the most dependent areas of the trunk. This phenomenon, according to the authors, is selflimited, causes only mild discomfort, and has no longterm sequelae. ${ }^{4}$

In our case, there was no history or clinical evidence of venous or lymphatic occlusion, peripheral edema, trauma, or local allergic reaction that could justify the development of vulvar edema.

Paracentesis is the most important factor in management of patients with moderate or severe OHSS as the presence of tense ascites accompanied by symptoms and hemodynamic changes are defining features for more advanced stages of disease. The edema was evident bilaterally within 24 hours of transvaginal paracentesis. This fact could justify, in our opinion, the correlation between the paracentesis and the development of vulvar edema. $^{6}$

We believe that the puncturing needle created a fistulous tract between the peritoneal cavity and subcutaneous tissues through which the ascitic fluid was forced, by the increased intraabdominal pressure, into the subcutaneous tissues, the most dependant area presenting as vulval edema. This phenomenon is self-limited and causes only mild discomfort without late sequelae. ${ }^{3}$ A similar mechanism has been suggested as the cause of postparacentesis scrotal edema in cirrhotic men with ascites. ${ }^{7}$ A similar experience was reported in cirrhotic women as well. ${ }^{8}$ 
It seems that this complication is not common in OHSS. Regarding the pathogenesis, we believe that our case further strengthens the previously suggested hypothesis that, paracentesis is the cause of vulvar edema. In cases in which paracentesis was not performed, the development of vulvar edema could probably be attributed to a patent canal of Nuck. Perhaps, to avoid this complication, it would be preferable to use either one of the upper abdominal regions as the puncturing site.

Funding: No funding sources

Conflict of interest: None declared

Ethical approval: Not required

\section{REFERENCES}

1. Bracero LA, Didomenico A. Massive vulval edema complicating preeclampsia: a management dilemma. J Perinatol. 1991;11:122.

2. Brittain C, Carlson JW, Gehlbach DL, Robertson AW. A case report of massive vulvar edema during tocolysis of preterm labor. Am J Obstet Gynecol. 1991;165:420-2.

3. Coccia ME, Bracco GL, Cattaneo A, Scarselli G. Massive vulvar edema in ovarian hyper stimulation syndrome. A case report. J Reprod Med. 1995;40(9):659-60.

4. Cline DL. Massive vulvar edema in ovarian hyperstimulation syndrome. J Reprod Med. 1996;41:780.

5. Luxman D, Cohen JR, Gordon D, Wolman I, Wolf Y, David MP. Unilateral vulvar edema associated with paracentesis in patients with severe ovarian hyperstimulation syndrome. A report of nine cases. J Reprod Med. 1996;41:771-4.

6. Vavilis D, Tzitzimikas S, Agorastos T, Loufopoulos A, Tsalikis T, Bontis JN. Postparacentesis bilateral massive vulvar edema in a patient with severe ovarian hyperstimulation syndrome. Fertil Steril. 2002;77:841-3.

7. Conn HO. Sudden scrotal edema in cirrhosis: a postparacentesis syndrome. Ann Intern Med. 1971;74:943-5.

8. Marks JW, Weil F. Conn's sudden labial edema. Ann Intern Med. 1971;75:810.

Cite this article as: Bhairavi S, Rajendran J, Dash S, Dash S. Postparacentesis vulvar edema in ovarian hyper stimulation syndrome. Int J Reprod Contracept Obstet Gynecol 2016;5:4064-6. 